\section{Gravity-Insensitive Flexure Pivot Oscillators}

\section{H. Kahrobaiyan ${ }^{1}$}

Instant-Lab,

École Polytechnique Fédérale de Lausanne (EPFL),

Microcity, Rue le la Maladière 71b,

Neuchâtel CH-2000, Switzerland

e-mail: mohammad.kahrobaiyan@epfl.ch

\section{E. Thalmann}

Instant-Lab,

École Polytechnique Fédérale de Lausanne (EPFL),

Microcity, Rue le la Maladière 71b,

Neuchâtel CH-2000, Switzerland

e-mail: etienne.thalmann@epfl.ch

\section{Rubbert}

INSA de Strasbourg,

Université de Strasbourg,

24 Bld de la Victoire,

Strasbourg 67084, France

e-mail: lennart.rubbert@insa-strasbourg.fr

\section{Vardi}

Instant-Lab, École Polytechnique Fédérale de Lausanne (EPFL), Microcity, Rue le la Maladière 71b,

Neuchâtel CH-2000, Switzerland

e-mail: ilan.vardi@epfl.ch

\section{S. Henein}

Instant-Lab,

École Polytechnique Fédérale de Lausanne (EPFL),

Microcity, Rue le la Maladière 71b,

Neuchâtel CH-2000, Switzerland

e-mail: simon.henein@epfl.ch

Classical mechanical watch plain bearing pivots have frictional losses limiting the quality factor of the hairspring-balance wheel oscillator. Replacement by flexure pivots leads to a drastic reduction in friction and an order of magnitude increase in quality factor. However, flexure pivots have drawbacks including gravity sensitivity, nonlinearity, and limited stroke. This paper analyzes these issues in the case of the cross-spring flexure pivot (CSFP) and presents an improved version addressing them. We first show that the cross-spring pivot cannot be simultaneously linear, insensitive to gravity, and have a long stroke: the $10 \mathrm{ppm}$ accuracy required for mechanical watches holds independently of orientation with respect to gravity only when the leaf springs cross at $12.7 \%$ of their length. But in this case, the pivot is nonlinear and the stroke is only $30 \%$ of the symmetrical (50\% crossing) crossspring pivot's stroke. The symmetrical pivot is also unsatisfactory as its gravity sensitivity is of order $10^{4} \mathrm{ppm}$. This paper introduces the codifferential concept which we show is gravity-insensitive. It is used to construct a gravity-insensitive flexure pivot (GIFP)

${ }^{1}$ Corresponding author.

Contributed by the Design Innovation and Devices of ASME for publication in the Journal of Mechanical Design. Manuscript received June 9, 2017; final manuscript received March 28, 2018; published online May 11, 2018. Assoc. Editor: Oscar Altuzarra. consisting of a main rigid body, two codifferentials, and a torsional beam. We show that this novel pivot achieves linearity or the maximum stroke of symmetrical pivots while retaining gravity insensitivity. [DOI: 10.1115/1.4039887]

\section{Introduction and Statement of Results}

1.1 Mechanical Watch Oscillators. The time base used in all mechanical watches is a harmonic oscillator consisting of a spiral spring attached to a balance wheel having a rigid pivot rotating on jeweled bearings, see Fig. 1(a). The pivoting motion on bearings causes significant friction and decreases watch autonomy. It also lowers oscillator quality factor to order 100 , this quantity is believed to be the most significant indicator of chronometric performance [3]. It is well known that flexure pivots drastically reduce friction, see Refs. [4] and [5], so flexure-pivot-based oscillators could improve mechanical watch time bases. In 2014, a flexure pivot was first used as a mechanical watch time base, see Fig. 1(b), thereby increasing quality factor to several thousand and watch autonomy by an order of magnitude to approximately 30 days [2]. This flexure pivot was the cross-spring flexure pivot (CSFP) first described by Wittrick [6]. CSFP consists of a rigid body attached to the ground by two perpendicular leaf-spring beams, see Fig. 2. In this watchmaking application [2], a special geometry [7] was chosen to minimize the effect of gravity on stiffness.

In this paper, we design a new flexure pivot which we name, the gravity-insensitive flexure pivot (GIFP), illustrated in Fig. 3. The design is based on the codifferential concept described in Sec. 4.1 which minimizes the effect of gravity on stiffness. This allows us to have zero nonlinearity of the torque-angle relationship or long angular stroke, while retaining a minimized effect of gravity on the stiffness of the pivot, making it desirable as a time base for mechanical watches.

Some of these results were announced in a lecture given by the first author [8].

1.2 Specifications of Flexure Pivots for Watch Oscillators. Mechanical harmonic oscillators must obey Hooke's law, which means that spring stiffness should be constant. Chronometric performance is equivalent to having as constant a frequency as possible, and since this depends on spring stiffness, portable timekeepers such as watches must have oscillators whose spring stiffness is insensitive to outside influences such as temperature and the orientation of the force of gravity. Since mechanical watches are only precise to within a few seconds per day, we will consider an effect to be negligible if it is of order $10 \mathrm{ppm}$ (parts per million), in watchmaking terms, about a $1 \mathrm{~s}$ per day accuracy.

In addition to being rotational bearings, flexure pivots provide an elastic restoring torque that can be used as springs for harmonic oscillators. However, their application to time bases can be limited by the following factors:

Limitation 1: Spring stiffness can be affected by the orientation of gravity load.

Limitation 2: Spring restoring torque can be a nonlinear function of rotation angle leading to an isochronism defect.

Limitation 3: Limited stroke makes it difficult to maintain and count oscillations using classical watch escapements.

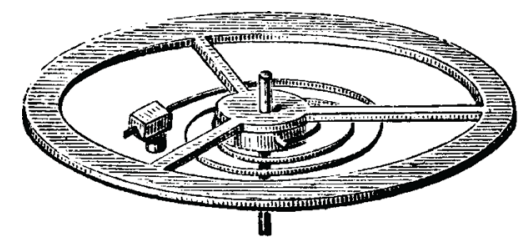

(a)

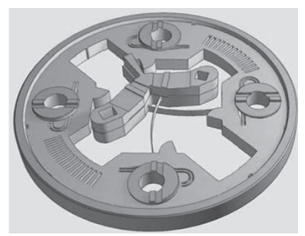

(b)
Fig. 1 (a) Rigid pivot watch time base [1] and (b) flexure pivot watch time base [2] 


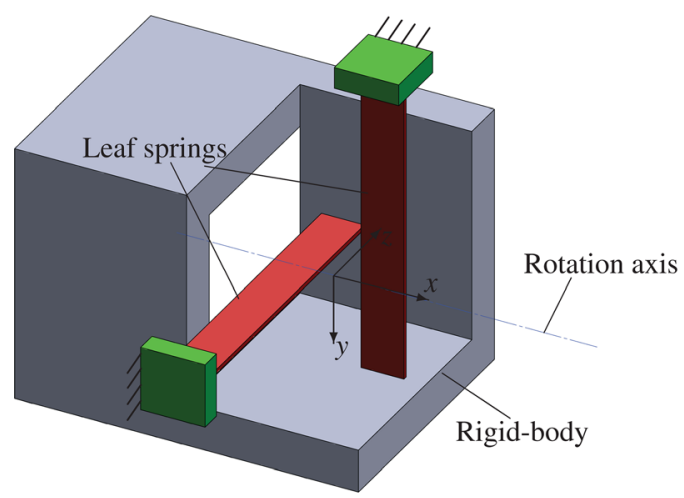

(a)
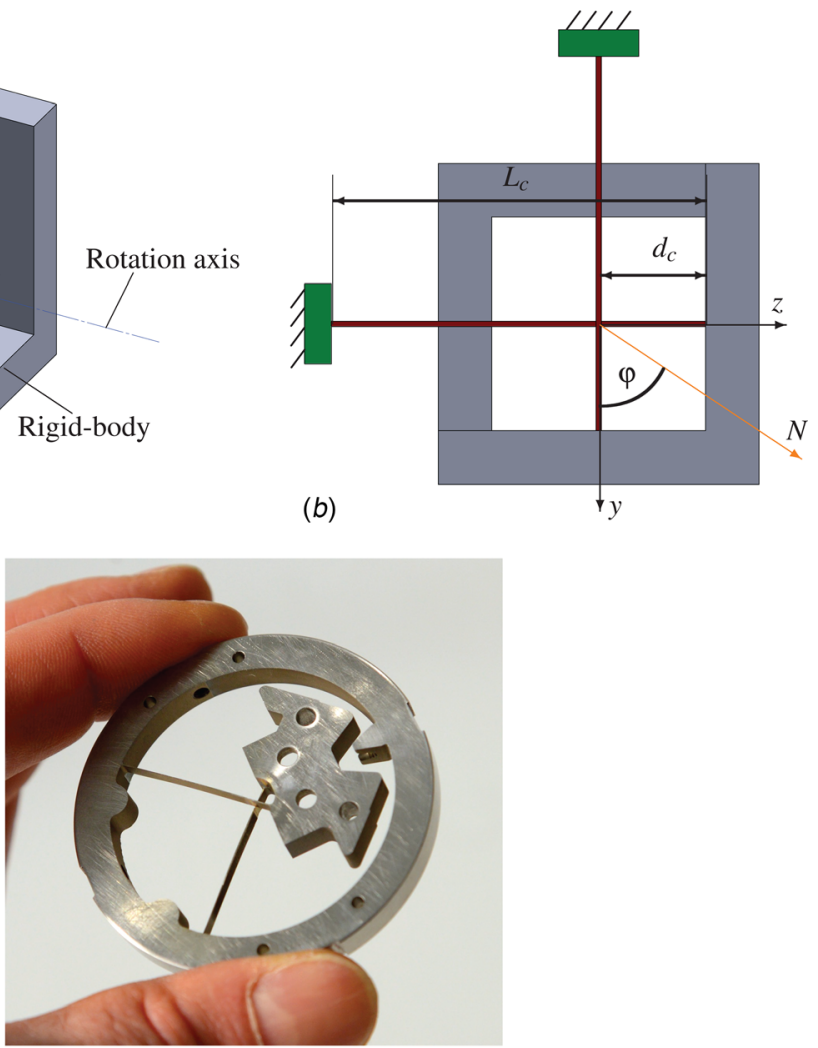

(c)

Fig. 2 CSFP: (a) three-dimensional view, (b) top view, and (c) demonstrator

Limitation 4: By construction, the kinematics of flexure pivots closely approximate rotational motion around a fixed axis but small translation can occur as angular rotation increases, a socalled parasitic shift.

We investigate the gravity sensitivity and nonlinearity of the torque-angle relationship as well as the stroke for a given aspect ratio and admissible stress level of the flexure pivot beams. The occurrence of a parasitic shift of the flexure pivot center is well known, see Ref. [9] for a detailed study.

In watchmaking, flexure pivot oscillators can have significantly higher frequency than classical mechanical oscillators, complicating the application of an escapement. For this reason, it is desirable to have flexure pivots with low rotational stiffness and lower oscillation frequency but this can lead to a problematic decrease in off-axis stiffness. This issue can be addressed by lattice flexures introduced in Ref. [10] since they have a higher ratio of off-axis stiffness to motion-direction stiffness as compared to blade-based flexures.

DEFINITION. We define gravity sensitivity as the relative change in rotational stiffness of a flexure pivot caused by gravity load. For a pivot with nominal stiffness $k_{0}$ (without load) and stiffness $k$ under gravity load, the gravity sensitivity in ppm is defined by

$$
\varepsilon=\left|\frac{k-k_{0}}{k_{0}}\right| \times 10^{6}
$$

We apply the term gravity-insensitive to an oscillator if its gravity sensitivity is of order $10 \mathrm{ppm}$. Otherwise, we will say that it is gravity-sensitive, see Sec. 5.1 for details.

Definition. We call a flexure pivot linear when its rotational stiffness is constant with respect to angular amplitude (its torque-angle relationship is linear). Otherwise, we call it nonlinear and we define relative nonlinearity to be the relative deviation of the rotational stiffness from the nominal value. We assume the range of motion of the pivot to be $-10 \mathrm{deg} \leq \theta \leq+10 \mathrm{deg}$. In this range, stiffness can be expressed by a power series with first two terms $k=k_{0}+k_{2} \theta^{2}+\mathcal{O}\left(\theta^{4}\right)$, where the higher order terms are negligible. The relative nonlinearity is defined to be

$$
\mu=\frac{k_{2}}{k_{0}}
$$

see Sec. 5.2 for details.

Definition. We define stroke as the rotation angle of the pivot which yields the maximum stress level in the beams, the so-called admissible value $\sigma_{\text {adm }}[11]$. We assume that the value of $\sigma_{\text {adm }}$ is the same for all pivots considered in this paper. Stroke is essentially the maximum amplitude of the oscillator. A formula for the stroke of flexure pivots is derived in Sec. 5.3.

We can now define goals for our flexure pivots.

Goal 1: Gravity insensitivity.

Goal 2: Minimal nonlinearity.

Goal 3: Maximal stroke for a given beam aspect ratio, admissible stress, and Young's modulus (material properties).

Goal 4: Minimal parasitic shift of the pivot axis.

Remark 1.2.1. Gravity sensitivity of flexure pivots is caused by the work of gravity load acting along the parasitic shift of the center of gravity. Hence, by minimizing parasitic shift we also minimize gravity sensitivity, and conversely. We, therefore, limit ourselves to the first three goals, keeping in mind that achieving goal 1 also achieves goal 4 .

Remark 1.2.2. Since the principal linear force affecting the chronometric performance of a portable timekeeper is gravity, we will use the term gravity to refer to all linear accelerations. The results of this paper apply to all linear accelerations.

\subsection{Statement of Results}

1.3.1 The Cross-Spring Flexure Pivot. The performance of CSFP is evaluated by varying the parameter $\delta_{c}=d_{c} / L_{c}$, where $L_{c}$ 
Table 1 Properties of CSFP resulting from finite element analysis (FEA) for special values of $\delta_{c}=d d L_{c}$

\begin{tabular}{|c|c|c|c|}
\hline Specification $\delta_{c}$ & 0.1 & 0.127 & 0.5 \\
\hline Gravity sensitivity (ppm) & $10^{3}$ & 10 & $10^{4}$ \\
\hline Normalized stroke $^{\mathrm{a}}(\%)$ & 29 & 30 & 86 \\
\hline Relative nonlinearity $\left(\mathrm{rad}^{-2}\right)$ & 0.00 & 0.02 & 0.16 \\
\hline
\end{tabular}

${ }^{\text {a }}$ We define normalized stroke to be the stroke divided by the maximum theoretical stroke given by Eq. (14) for $\delta=0.5$.

is the length of the beams and $d_{c}$ denotes the distance between their crossing and the mobile end, as shown in Fig. 2(b).

Table 1 gives results for special values of $\delta_{c}$ satisfying the goals of Sec. 1.2. For $\delta_{c}=0.1$, relative nonlinearity is minimized, for $\delta_{c}=0.127$ gravity sensitivity is minimized (as well as parasitic shift, see Ref. [6]) and choosing $\delta_{c}=0.5$ maximizes the stroke (gravity sensitivity, stroke, and relative nonlinearity are defined in Sec. 1.2). Choosing $\delta_{c}=0.127$ minimizes gravity sensitivity to order $10 \mathrm{ppm}$ but stroke is only $30 \%$ of the maximum theoretical stroke occurring at $\delta_{c}=0.5$ and the relative nonlinearity is nonzero.

1.3.2 New Gravity-Insensitive Flexure Pivot. The performance of GIFP is evaluated by varying $\delta_{g}=d_{g} / L_{g}$ which is the equivalent of $\delta_{c}$ for this pivot, with $d_{g}$ and $L_{g}$ as shown in Fig. 3(a). Table 2 summarizes the performance of GIFP for special values of $\delta_{g}$ corresponding to those in Table 1 . In particular, $\delta_{g}=0.5$ gives a long-stroke GIFP meeting goals 1 and 3 of
Table 2 Properties of GIFP resulting from FEA for special values of $\delta_{g}=d_{g} / L_{g}$

\begin{tabular}{lccc}
\hline \hline Specification & & & \\
\hline Gravity sensitivity (ppm) & 0.1 & 0.127 & 0.5 \\
Normalized stroke $(\%)$ & 10 & 10 & 10 \\
Relative nonlinearity $\left(\mathrm{rad}^{-2}\right)$ & 29 & 30 & 86 \\
\hline \hline
\end{tabular}

${ }^{a}$ We define normalized stroke to be the stroke divided by the maximum theoretical stroke given by Eq. (14) for $\delta=0.5$.

Sec. 1.2. Choosing $\delta_{g}=0.1$ gives a linear GIFP meeting goals 1 and 2 of Sec. 1.2.

1.3.3 Comparison Between the New and the Conventional Flexure Pivots. Table 2 shows that GIFP gravity sensitivity is of order $10 \mathrm{ppm}$ for all values of $\delta_{g}=d_{g} / L_{g}$. So, GIFP achieves goal 1 of the paper given in Sec. 1.2, for all values of $\delta_{g}$. By comparison, Table 1 shows that CSFP gravity sensitivity changes from order $10 \mathrm{ppm}$ to order $10^{4} \mathrm{ppm}$, depending on the value of $\delta_{c}$.

The relative nonlinearity of both CSFP and GIFP becomes zero at $\delta_{c}=\delta_{g}=0.1$, achieving goal 2 of the paper. For this value, CSFP gravity sensitivity is of order $10^{3} \mathrm{ppm}$ whereas GIFP gravity sensitivity is of order $10 \mathrm{ppm}$, meaning that only GIFP can achieve both goal 1 and goal 2 .

In terms of stroke, the special value $\delta_{c}=\delta_{g}=0.5$ leads to maximum stroke for both pivots, achieving goal 3. For this value, CSFP gravity sensitivity is of order $10^{4} \mathrm{ppm}$ whereas GIFP gravity sensitivity is of order $10 \mathrm{ppm}$, meaning that only GIFP can achieve both goal 1 and goal 3 .

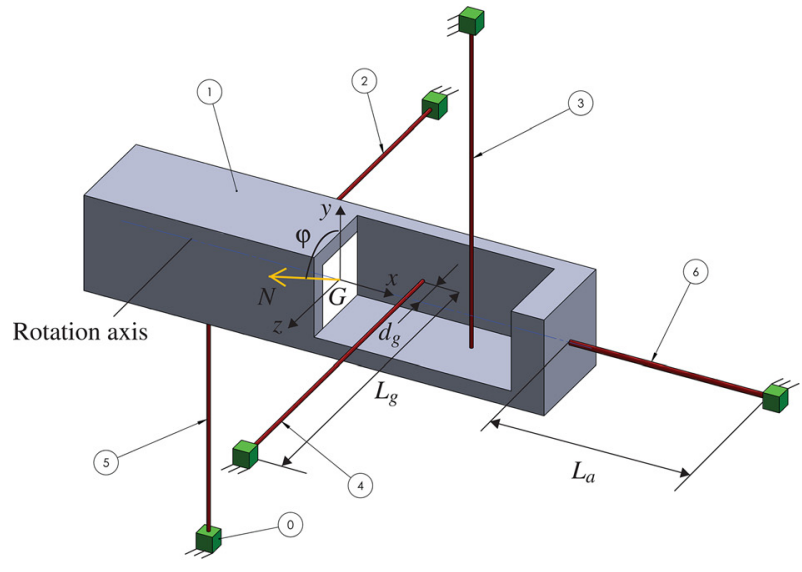

(a)

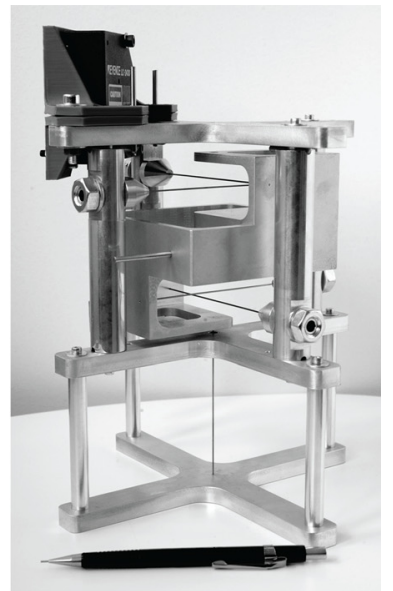

(b)

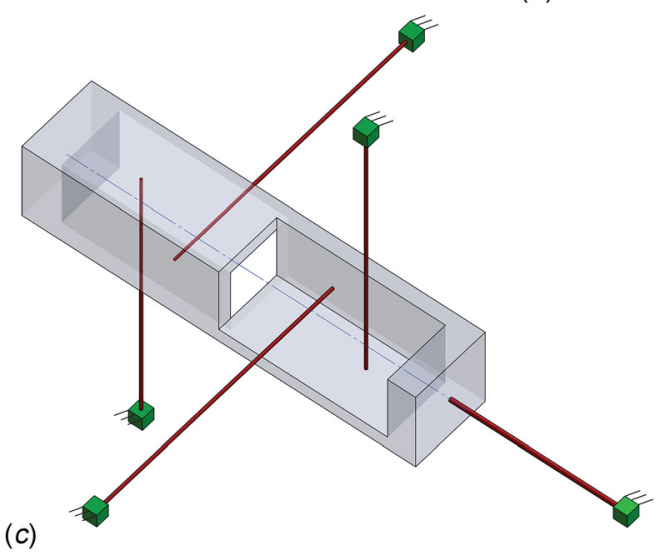

Fig. 3 New GIFP: (a) modelization, (b) demonstrator, and (c) transparent view 
Table 3 Comparison of CSFP with $\delta_{c}=0.127$, GIFP with $\delta_{g}=0.1$, and GIFP with $\delta_{g}=0.5$ using FEA results

\begin{tabular}{lccc}
\hline \hline Specification & CSFP & GIFP & GIFP \\
& $\delta_{c}=0.127$ & $\delta_{g}=0.1$ & $\delta_{g}=0.5$ \\
\hline Gravity sensitivity (ppm) & 10 & 10 & 10 \\
Normalized stroke (\%) & 31 & 29 & 86 \\
Relative nonlinearity $\left(\mathrm{rad}^{-2}\right)$ & 0.02 & 0.00 & 0.22 \\
\hline \hline
\end{tabular}

Table 3 compares GIFP with $\delta_{g}=0.5$ or $\delta_{g}=0.1$ and CSFP with $\delta_{c}=0.127$ and shows that GIFP is superior to CSFP in terms of stroke or linearity while having the same order of gravity insensitivity. The stroke of GIFP with $\delta_{g}=0.5$ is 2.9 times that of CSFP with $\delta_{c}=0.127$, the only value providing gravity insensitivity for CSFP.

\section{Plan of Paper}

Our study of suitable flexure pivots begins with an analysis of the CSFP, which has already been used as a watch oscillator. We then introduce a new flexure pivot which we name, the GIFP. This pivot aims at improving gravity insensitivity over the conventional pivot.

In Sec. 3, we derive the rotational stiffness of the CSFP. We characterize it for small loads and comment on the influence of the geometric parameter $\delta_{c}$.

In Sec. 4, we explain the design of the new GIFP and derive its rotational stiffness. Its design is based on the concept of the codifferential described in Sec. 4.1, which we show is insensitive to gravity in Sec. 4.2. We explain that the design has only one degree-of-freedom (DOF), a rotation, and is statically determinate (isostatic), a desirable feature.

In Sec. 5, we compare how CSFP and GIFP satisfy the goals given in Sec. 1.2. Section 5.1 analyses the gravity sensitivity of both pivots which will be necessary to reach goal 1. In Sec. 5.2, we give formulas for the relative nonlinearity of CSFP and GIFP. This will be used to reach goal 2. Finally, Sec. 5.3 provides explicit formulas for the stroke of pivots in order to analyze goal 3.

All our analytical results were validated using FEA. CSFP and GIFP were modeled in ANSYS ${ }^{\circledR}$ wORKBENCH, Release 18.1 (ANSYS, Canonsburg, PA) [12] using a mesh of 40 beam elements for each flexible beam.

\section{Analysis of the Cross-Spring Flexure Pivot}

The CSFP beam geometry is such that it has one DOF, a rotation with axis lying on the intersection of the beams midplanes [11], the $x$-axis in Fig. 2(a).

In this section, we derive the rotational stiffness of CSFP. The coordinate system of the pivot is $x, y, z$ in Fig. 2. Since the out-of- plane bending stiffness of the leaf-spring beams of CSFP is much higher than their in-plane ( $y-z$ plane) bending stiffness, we only consider in-plane mechanical behavior and assume no out-ofplane motion. Since the in-plane translational degrees-of-freedom (translations along the $y$ - and $z$-directions) are blocked by the beams, the only DOF of CSFP is rotation about an axis perpendicular to the $y-z$ plane. For infinitesimal rotation angles, the rotation axis intersects the $y-z$ plane at the crossing point of the beams. However, as rotation angle increases, a translation of the rotation axis along the $y-z$ plane occurs, the so-called parasitic shift.

The center of mass is assumed to be located on the axis of rotation. The distance between the crossing point and the mobile extremities of the beams is denoted by $d_{c}$ and the length of the beams is denoted by $L_{c}$, see Fig. 2. The geometric parameter $\delta_{c}=d_{c} / L_{c}$ has a direct influence on gravity sensitivity, stroke, nonlinearity, and parasitic shift of CSFP. The area moment of inertia of the beams is denoted by $I_{c}$.

Remark 3. The geometric parameter $\delta_{c}$ of CSFP has range $0 \leq$ $\delta_{c} \leq 1$, but due to symmetry, we analyze the behavior of the pivot in the range $0 \leq \delta_{c} \leq 0.5$. To analyze CSFP for $0.5<\delta_{c} \leq 1$, one can use the results presented here with $\delta_{c}^{\prime}=1-\delta_{c}$ due to interchangeability of the rotating rigid body and the fixed frame.

3.1 Rotational Stiffness. Figure 4 shows a flexure pivot subject to a rotation of angle $\theta$. In the end position, the center of rotation is located at the crossing point $O^{\prime}$ of the tangents to the beams at their mobile extremities. The rotation axis has thus shifted from $O$ to $O^{\prime}$ and the vector $\mathbf{O O}^{\prime}$ denotes the parasitic shift.

Each beam of CSFP can be modeled as a cantilever beam of length $L$ under an axial load $P$ with lateral deflection $f$ and slope $\theta$ at its mobile extremity, see Fig. 5. For such beam, EulerBernoulli beam theory gives the following force-displacement relationship, see Refs. [13] and [14]:

$$
\left(\begin{array}{c}
F L^{2} / \mathrm{EI} \\
M L / \mathrm{EI}
\end{array}\right)=\mathbf{K}\left(\begin{array}{c}
f / L \\
\theta
\end{array}\right)
$$

where $M$ and $F$ denote the bending moment and shear force at the mobile extremity of the cantilever beam, $E$ is Young's modulus of the beam, and $I$ is area moment of inertia. $\mathbf{K}$ is the stiffness matrix

$$
\mathbf{K}=\frac{\rho}{2(1-\cosh \rho)+\rho \sinh \rho}\left(\begin{array}{cc}
\rho^{2} \sinh \rho & \rho(1-\cosh \rho) \\
\rho(1-\cosh \rho) & \rho \cosh \rho-\sinh \rho
\end{array}\right)
$$

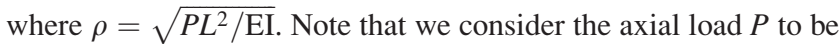
positive when it is tensile and negative when it is compressive.

We derive CSFP rotational stiffness $k_{c}$ by dividing the restoring torque about point $O^{\prime}$ by the rotation angle $\theta$, see Fig. 4. CSFP rotational stiffness is

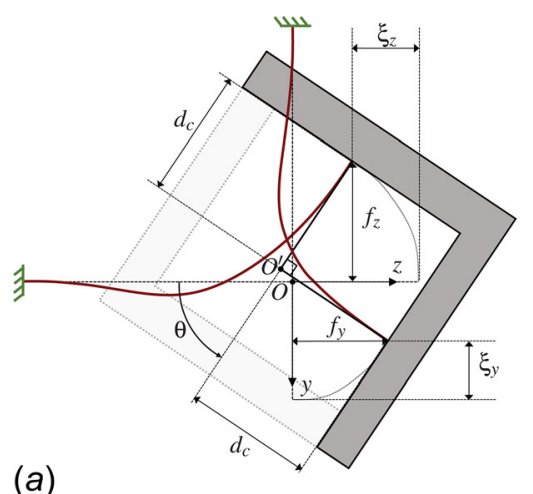

(a)

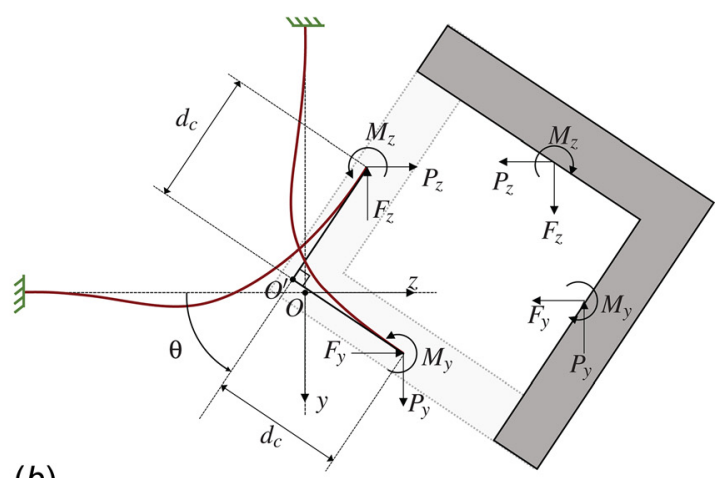

(b)

Fig. 4 A CSFP subjected to a rotation of angle $\theta$ around axis $x$. (a) Deflections of the beams and $(b)$ an exploded view to indicate reaction forces and moments of the beams. 


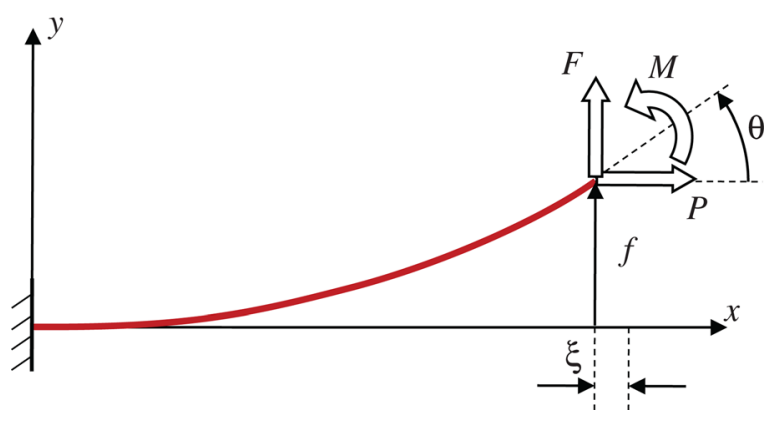

Fig. 5 A cantilever beam under axial load $P$, shear force $F$, and bending moment $\boldsymbol{M}$ with axial shortening $\xi$, lateral deflection $f$, and slope $\theta$ at its mobile extremity

$$
k_{c}=\left[M_{y}+M_{z}+\left(F_{y}+F_{z}\right) d_{c} \cos \theta-\left(P_{y}+P_{z}\right) d_{c} \sin \theta\right] / \theta
$$

where the subscripts $y$ and $z$ refer to the beams initially lying on the $y$ - and $z$-axes. The lateral deflections of the beams depicted in Fig. 4 are

$$
f_{y}=d_{c} \sin \theta+\mathbf{O O}^{\prime} \cdot \mathbf{e}_{z}, \quad f_{z}=d_{c} \sin \theta-\mathbf{O O}^{\prime} \cdot \mathbf{e}_{y}
$$

where $\mathbf{e}_{y}$ and $\mathbf{e}_{z}$ denote the unit vectors of the $y$ - and $z$-axes, respectively. Since the parasitic shift is relatively small, the terms associated with the parasitic shift in Eq. (4) produce negligible restoring torques. Hence, we will assume that $f_{y}=f_{z}=d_{c} \sin \theta$ in Eq. (1). Note that this is a standard assumption in the analysis of flexure pivots, see Refs. [15] and [16]. With this assumption, substituting Eq. (1) in Eq. (3), we find the rotational stiffness to be

$k_{c}=\frac{E I_{c}}{L_{c}}\left[\left(\begin{array}{ll}\delta_{c} \cos \theta & 1\end{array}\right)\left(\mathbf{K}_{y}+\mathbf{K}_{z}\right)\left(\begin{array}{c}\delta_{c} \frac{\sin \theta}{\theta} \\ 1\end{array}\right)-\delta_{c}\left(\lambda_{y}+\lambda_{z}\right) \frac{\sin \theta}{\theta}\right]$

where $\lambda_{y}=P_{y} L_{c}^{2} / E I_{c}$ and $\lambda_{z}=P_{z} L_{c}^{2} / E I_{c}$ are the normalized axial loads of the beams in the $y$ - and $z$-directions. In addition, $\mathbf{K}_{y}$ and $\mathbf{K}_{z}$ can be calculated from Eq. (2) using $\rho=\sqrt{\lambda_{y}}$ and $\rho=\sqrt{\lambda_{z}}$, respectively.

When the pivot is not rotated $(\theta=0)$, the shear forces are zero $\left(F_{y}=F_{z}=0\right)$ and the axial loads of the beams are

$$
P_{y}=N \cos \varphi, \quad P_{z}=N \sin \varphi
$$

where $N$ is the load on the main body, generally assumed to be gravity, and $\varphi$ is the angle between the load and the $y$-axis ( $0 \mathrm{deg}$ $\leq \varphi<360 \mathrm{deg}$ ), see Fig. 2.

Rotation of the pivot gives rise to shear forces and leads to slight changes in axial loads during oscillation. We neglect this effect and assume that Eq. (6) holds for the entire oscillation.

3.1.1 Approximation by Series Expansion. In order to characterize the behavior of small loads, we use the series expansion of the hyperbolic functions in Eq. (2) to obtain the following formulas for the stiffness matrix:

$$
\mathbf{K}=\mathbf{K}_{0}+\frac{\lambda}{30}\left(\begin{array}{cc}
36 & -3 \\
-3 & 4
\end{array}\right)-\frac{\lambda^{2}}{12,600}\left(\begin{array}{cc}
18 & -9 \\
-9 & 22
\end{array}\right)+\mathcal{O}\left(\lambda^{3}\right)
$$

where $\lambda=P L^{2} / E I$ is the normalized axial load, $\mathcal{O}$ denotes the Landau notation [17], and $\mathbf{K}_{0}$ is the stiffness matrix of a cantilever beam undergoing no axial load

$$
\mathbf{K}_{0}=\left(\begin{array}{cc}
12 & -6 \\
-6 & 4
\end{array}\right)
$$

Substituting Eq. (7) in Eq. (5) and using the series expansion of trigonometric functions around $\theta=0$, we arrive at the following formula for the rotational stiffness of CSFP:

$$
\begin{aligned}
k_{c}= & k_{c, 0}+\frac{2 E I_{c}}{15 L_{c}}\left(9 \delta_{c}^{2}-9 \delta_{c}+1\right) \bar{N}_{c}(\sin \varphi+\cos \varphi) \\
& -\frac{E I_{c}}{6300 L_{c}}\left(9 \delta_{c}^{2}-9 \delta_{c}+11\right) \bar{N}_{c}^{2}+\frac{8 E I_{c}}{L_{c}}\left(\delta_{c}-2 \delta_{c}^{2}\right) \theta^{2} \\
& -\frac{E I_{c}}{30 L_{c}}\left(24 \delta_{c}^{2}-7 \delta_{c}\right) \theta^{2} \bar{N}_{c}(\sin \varphi+\cos \varphi) \\
& +\frac{E I_{c}}{2100 L_{c}}\left(2 \delta_{c}^{2}-\delta_{c}\right) \theta^{2} \bar{N}_{c}^{2}+\mathcal{O}\left(\theta^{4}\right)+\mathcal{O}\left(\bar{N}_{c}^{3}\right)
\end{aligned}
$$

where $\bar{N}_{c}$ represents the normalized load exerted on the flexure pivot $\bar{N}_{c}=N L_{c}^{2} / E I_{c}$. In addition, $k_{c, 0}$ denotes the nominal stiffness of the pivot for an infinitesimal rotation in the absence of gravity, i.e., $\theta=0$ and $\bar{N}_{c}=0$

$$
k_{c, 0}=2 \frac{E I_{c}}{L_{c}}\left(\begin{array}{ll}
\delta_{c} & 1
\end{array}\right) \mathbf{K}_{0}\left(\begin{array}{c}
\delta_{c} \\
1
\end{array}\right)=\frac{8 E I_{c}}{L_{c}}\left(3 \delta_{c}^{2}-3 \delta_{c}+1\right)
$$

The normalized nominal stiffness of CSFP $\bar{k}_{c, 0}=k_{c, 0} L_{c} / E I_{c}$ is computed analytically and using FEA, and illustrated in Fig. 6. The figure indicates a good match between the analytical and the FEA results.

Remark 3.1.1.1. $\delta_{c}=(1 / 2)-(\sqrt{5} / 6)=0.127$ satisfying the equation $9 \delta_{c}^{2}-9 \delta_{c}+1=0$ minimizes the effect of load (gravity) on rotational stiffness.

Remark 3.1.1.2. $\delta_{c}=0.5$ minimizes the nominal stiffness $k_{c, 0}$.

Remark 3.1.1.3. At $\varphi=45 \mathrm{deg}$ and $\varphi=225 \mathrm{deg}$, the effect of gravity load $N$ on the rotational stiffness is maximum.

\section{Design and Analysis of the Gravity-Insensitive Flexure Pivot}

The GIFP illustrated in Fig. 3(a) will be completely described in this section. This mechanism is based on a new flexure element we have called the codifferential.

4.1 The Codifferential. We define the codifferential to be the flexure element consisting of two identical beams subjected to the same kinematic boundary conditions placed in such way that their axial loads (due to gravity) are opposite (same magnitude but opposite signs). In other words, when one beam is subjected to tensile axial load, the other one is subjected to compressive axial load of equal magnitude.

Figure 7(a) shows a codifferential consisting of two flexible beams of length $L$ positioned symmetrically at a 180 deg rotation in the $x-z$ plane with respect to point $G$, the center of gravity.

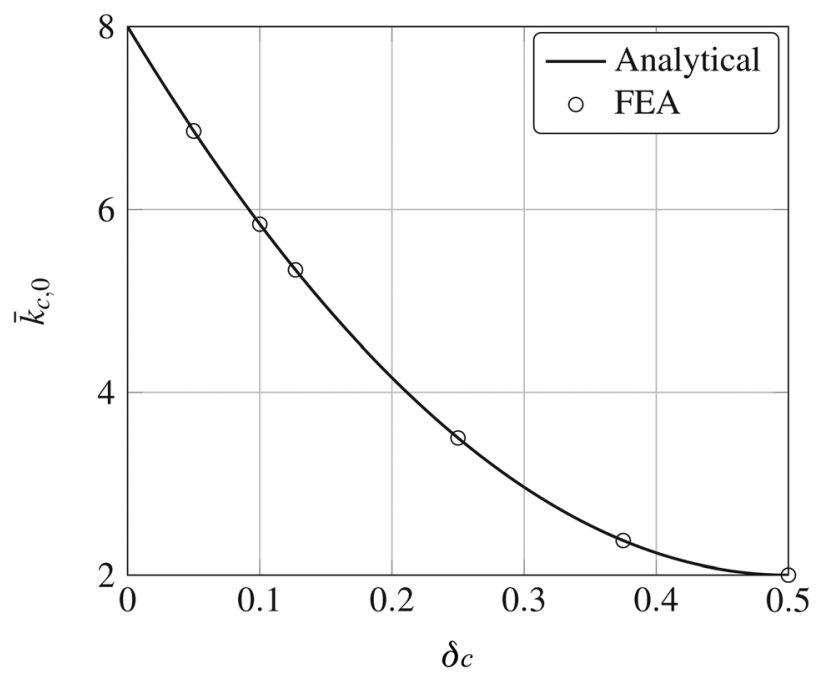

Fig. 6 Normalized nominal stiffness $\overline{\boldsymbol{k}}_{c, 0}$ of CSFP versus geometric parameter $\delta_{c}$. Analytical and FEA results are shown. 


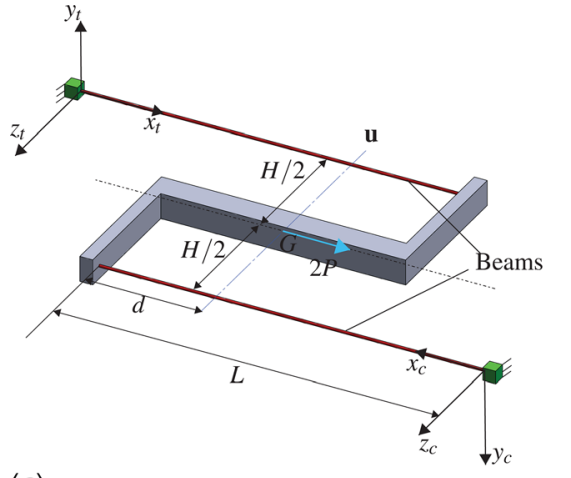

(a)

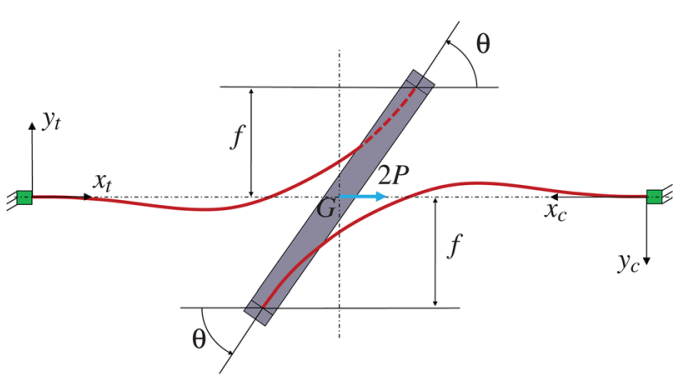

(b)

Fig. 7 The codifferential. (a) Three-dimensional view and (b) side view under rotation $\theta$ about the u-axis.

Both beams have the same geometric and material properties. The beam arrangement allows rotation around $\mathbf{u}$-axis, see Fig. 7(a).

The total axial load applied to the codifferential at point $G$ is denoted by $2 P$, as shown in Fig. $7(b)$. Since both beams are at distance $H / 2$ from point $G$, the axial load on each beam is $P$. One beam is under tension with coordinates at its base $x_{t}, y_{t}, z_{t}$, the other is under compression with coordinates at its base $x_{c}, y_{c}, z_{c}$.

In their initial position, both cantilever beams cross the rotation axis $\mathbf{u}$ at distance $d$ from their mobile end. They have the same kinematic boundary conditions at their mobile end: deflection $f$ and slope $\theta$, as shown in Fig. 7(b).

4.2 Codifferential Gravity Sensitivity. We quantify gravity sensitivity by considering the behavior of the stiffness matrix under axial load.

The geometry of the codifferential is such that when one beam is under tension, the other is under compression. We, therefore, compute the stiffness matrix of the codifferential $\mathbf{K}_{\mathrm{cd}}$ by adding the stiffness matrix $\mathbf{K}_{+}$of the beam under tension and the stiffness matrix $\mathbf{K}_{-}$of the beam under compression. Note that the stiffness matrix of a cantilever $\mathbf{K}$ is given in Eq. (7) as a function of the normalized axial load $\lambda=P L^{2} / E I$. Going from tension to compression changes the sign of $P$ and therefore the sign of $\lambda$, so that $\mathbf{K}_{-}(\lambda)=\mathbf{K}_{+}(-\lambda)$. Substituting these values in Eq. (7) and adding gives

$$
\mathbf{K}_{\mathrm{cd}}=\mathbf{K}_{+}+\mathbf{K}_{-}=2 \mathbf{K}_{0}-\frac{\lambda^{2}}{6300}\left(\begin{array}{cc}
18 & -9 \\
-9 & 22
\end{array}\right)+\mathcal{O}\left(\lambda^{3}\right)
$$

A cancelation of the $\lambda$ term occurs in Eq. (10) showing that the codifferential improves gravity insensitivity since the nonconstant term has gone from first-order to second-order.

4.3 Design. We use the codifferential to design our gravityinsensitive pivot. Since a pivot has one DOF and a rigid body has six degrees-of-freedom, the simplest design consists of a rigid main body and five beams blocking all but one DOF. The five beams consist of two codifferentials having two beams each and a fifth torsional beam insensitive to axial load. Note that the two codifferentials share the same rigid mass.

The configuration of GIFP is depicted in Fig. 3(a). The design comprises a rigid body (1) attached to the ground (0) by five beams: a codifferential in the $y$-direction with beams (3) and (5), a codifferential in the $z$-direction with beams (2) and (4), and a single torsional beam (6) in the $x$-direction. The single DOF is rotation around the $x$-axis.

The mechanism is statically determinate (isostatic) as there is only one DOF and there are no redundant constraints. The codifferential beams of length $L_{g}$ cross the axis of rotation at a distance $d_{g}$ from their mobile ends. The geometric parameter $\delta_{g}=d_{g} / L_{g}$ characterizes GIFP performance.

Remark 4.3. For GIFP, we vary $\delta_{g}$ from 0 to $0.5\left(0 \leq \delta_{g} \leq 0.5\right)$. To analyze a GIFP with $0.5<\delta_{g} \leq 1$, one can use the results for GIFP presented here with $\delta_{g}^{\prime}=1-\delta_{g}$ due to interchangeability of the rotating rigid body and the fixed frame.

4.4 Rotational Stiffness. Since axial load has no effect on the stiffness of torsional beam (6) of Fig. 3(a), the $x$-component of the gravity load has no effect on the rotational stiffness of the pivot. We, therefore, assume that the gravity load $N$ is applied in the $y-z$ plane at an angle $\varphi$ with respect to $y$-axis.

We use the method described in Sec. 3.1 to derive the following rotational stiffness of GIFP:

$$
\begin{aligned}
k_{g}= & k_{g, 0}+\frac{16 E I_{g}}{L_{g}}\left(\delta_{g}-2 \delta_{g}^{2}\right) \theta^{2}+\frac{E I_{g}}{4200 L_{g}}\left(2 \delta_{g}^{2}-\delta_{g}\right) \theta^{2} \bar{N}_{g}^{2} \\
& -\frac{E I_{g}}{12,600 L_{g}}\left(9 \delta_{g}^{2}-9 \delta_{g}+11\right) \bar{N}_{g}^{2}+\mathcal{O}\left(\theta^{2}\right)+\mathcal{O}\left(\bar{N}_{g}^{4}\right)
\end{aligned}
$$

where $E$ and $I_{g}$ are Young's modulus and the area moment of inertia of GIFP bending beams, $\bar{N}_{g}=N L_{g}^{2} / E I_{g}$ is the normalized gravity load, and $k_{g, 0}$ is the nominal stiffness of the pivot

$$
k_{g, 0}=k_{a}+\frac{16 E I_{g}}{L_{g}}\left(3 \delta_{g}^{2}-3 \delta_{g}+1\right)
$$

in which $k_{a}=G_{a} J_{a} / L_{a}$ is the torsional stiffness of the torsional beam of length $L_{a}$, shear modulus $G_{a}$, and polar area moment of inertia $J_{a}$.

The normalized nominal stiffness of GIFP $\bar{k}_{g, 0}=k_{g, 0} L_{g} / E I_{g}$ is computed analytically and using FEA, and illustrated in Fig. 8. The figure indicates a good match between the analytical and the FEA results.

Remark 4.4.1. The gravity insensitivity of GIFP is improved as compared to CSFP since there is no first-order effect of load $\bar{N}_{g}$ (gravity) on its rotational stiffness $k_{g}$, see Eq. (11). For CSFP, the first-order effect of load $\bar{N}_{c}$ on its rotational stiffness vanishes only when $\delta_{c}=0.127$, see Eq. (8).

Remark 4.4.2. Even when $\delta_{g}=\delta_{c}=0.127$, GIFP is gravityinsensitive for a wider range of rotational angles $\theta$ than for CSFP, since there is a $\theta^{2} \bar{N}_{c}$ term in Eq. (8) but no $\theta^{2} \bar{N}_{g}$ term in Eq. (11).

Remark 4.4.3. $\delta_{g}=0.5$ minimizes the nominal stiffness $k_{g, 0}$ of GIFP, see Eq. (12).

\section{Comparison Between Cross-Spring Flexure Pivot and Gravity-Insensitive Flexure Pivot}

Having analyzed both pivots independently and derived their rotational stiffness, we can now compare how they satisfy the goals defined in Sec. 1.2. 


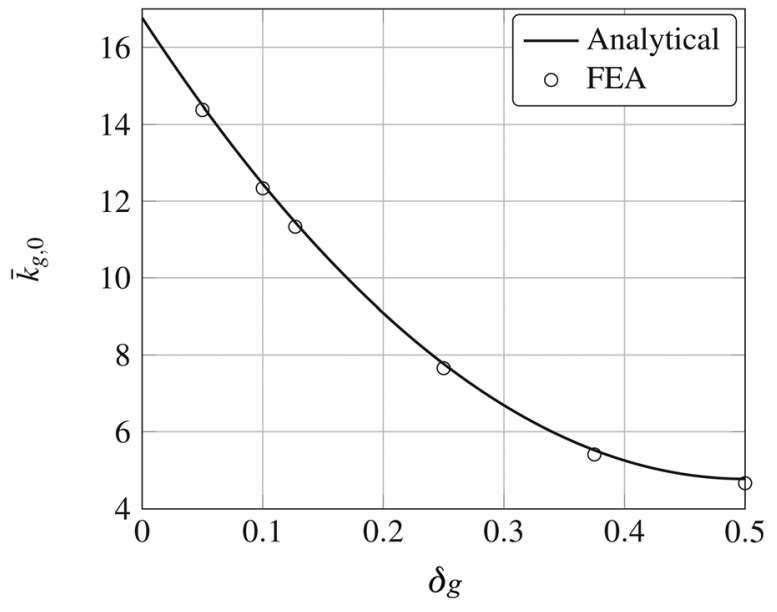

Fig. 8 Normalized nominal stiffness $\overline{\boldsymbol{k}}_{\boldsymbol{g}, 0}$ of GIFP versus geometric parameter $\delta_{g}$. Analytical and FEA results are shown.

5.1 Gravity Sensitivity. Here, we use the results of Secs. 3.1 and 4.4 to investigate the gravity sensitivity of CSPF and GIFP. We define the gravity sensitivity of CSFP $\varepsilon_{c}$ and the gravity sensitivity of GIFP $\varepsilon_{g}$ as

$$
\varepsilon_{c}=\left|\frac{k_{c}-k_{c, 0}}{k_{c, 0}}\right| \times 10^{6}, \quad \varepsilon_{g}=\left|\frac{k_{g}-k_{g, 0}}{k_{g, 0}}\right| \times 10^{6}
$$

Note that they are given in ppm. $\varepsilon_{c}$ and $\varepsilon_{g}$ are plotted in Fig. 9 versus the geometric parameters $\delta_{c}$ and $\delta_{g}$, respectively, for different normalized gravity loads and $\theta=0.1 \mathrm{deg}$. There is a good match between the analytical and the FEA results.

Figure $9(a)$ shows that $\varepsilon_{c}$ is very sensitive to the choice of $\delta_{c}$. It varies from the order of $10 \mathrm{ppm}$ for $\delta_{c}=0.127$ to the order of $10^{4}$ ppm for $\delta_{c}=0.5$. A CSFP is gravity-insensitive, achieving goal 1 only for $\delta_{c}=0.127$.

On the other hand, Fig. $9(b)$ shows that GIFP gravity sensitivity is much less affected by $\delta_{g}$ and is of order $10 \mathrm{ppm}$ for all values of the geometric parameter. Hence, GIFP satisfies goal 1 in a way that is relatively insensitive to the choice of $\delta_{g}$.

Figure 10 shows the gravity sensitivity of CSFP and GIFP versus the rotation angle $\theta$ for a normalized gravity load $\bar{N}_{g}=0.2$ and the geometric parameter $\delta_{g}=\delta_{c}=0.127$ which gives the minimum gravity sensitivity for CSFP. The figure shows that GIFP gravity sensitivity is almost constant with respect to $\theta$. However, the gravity sensitivity of CSFP increases with $\theta$. GIFP is gravityinsensitive for a wide range of $\theta$ but CSFP is gravity-insensitive only for small values of $\theta(\theta<8 \mathrm{deg})$. The reason for this is explained in Remark 4.4.2.

5.2 Nonlinearity. This section deals with stiffness nonlinearity with respect to rotation angle. We assume the range of motion of the pivots to be $-10 \mathrm{deg} \leq \theta \leq+10 \mathrm{deg}$. In this range, we express pivot stiffness by a series expansion with first two terms, where the higher order terms are negligible. Using Eqs. (8) and (11) under zero gravity load $(N=0)$, the series expansions of the CSFP and GIFP rotational stiffness are, respectively

$$
\begin{aligned}
& k_{c}=k_{c, 0}+\frac{8 E I_{c}}{L_{c}}\left(\delta_{c}-2 \delta_{c}^{2}\right) \theta^{2}+\mathcal{O}\left(\theta^{4}\right), \\
& k_{g}=k_{g, 0}+\frac{16 E I_{g}}{L_{g}}\left(\delta_{g}-2 \delta_{g}^{2}\right) \theta^{2}+\mathcal{O}\left(\theta^{4}\right)
\end{aligned}
$$

We define relative nonlinearity to be the ratio of the coefficient of $\theta^{2}$ to the nominal stiffness of the pivots, this normalization is useful for comparing nonlinearity of different pivots. Applying this to CSFP and GIFP, respectively, gives relative nonlinearities

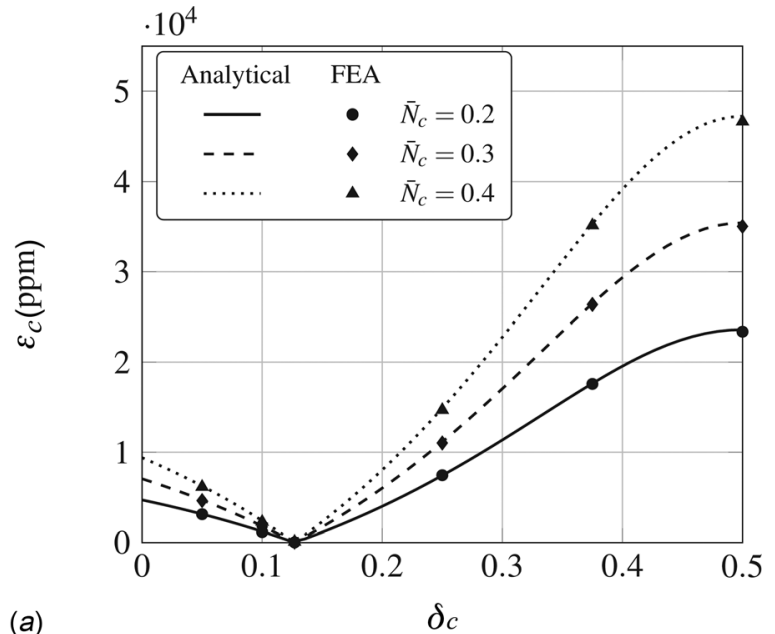

(a)

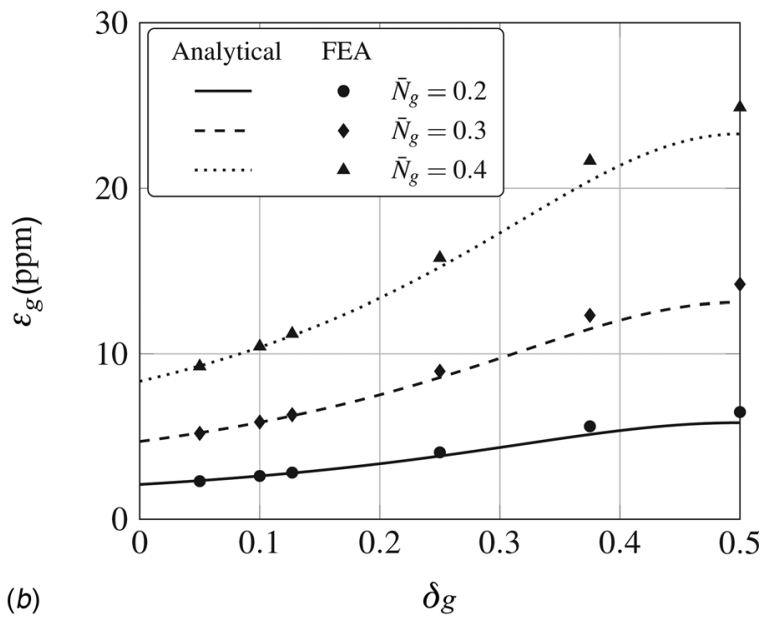

Fig. 9 (a) CSFP gravity sensitivity $\varepsilon_{c}$ versus $\delta_{c}=d_{d} L_{c}$ and (b) GIFP gravity sensitivity $\varepsilon_{g}$ versus $\delta_{g}=d_{g} L_{g}$ for different normalized gravity loads at an angle $\varphi=45 \mathrm{deg}$ and a rotation $\theta=0.1$ deg. Analytical and FEA results are shown.

$$
\mu_{c}=\frac{\delta_{c}-2 \delta_{c}^{2}}{3 \delta_{c}^{2}-3 \delta_{c}+1}, \quad \mu_{g}=\frac{\delta_{g}-2 \delta_{g}^{2}}{3 \delta_{g}^{2}-3 \delta_{g}+1+\frac{1}{16(1+\nu)}}
$$

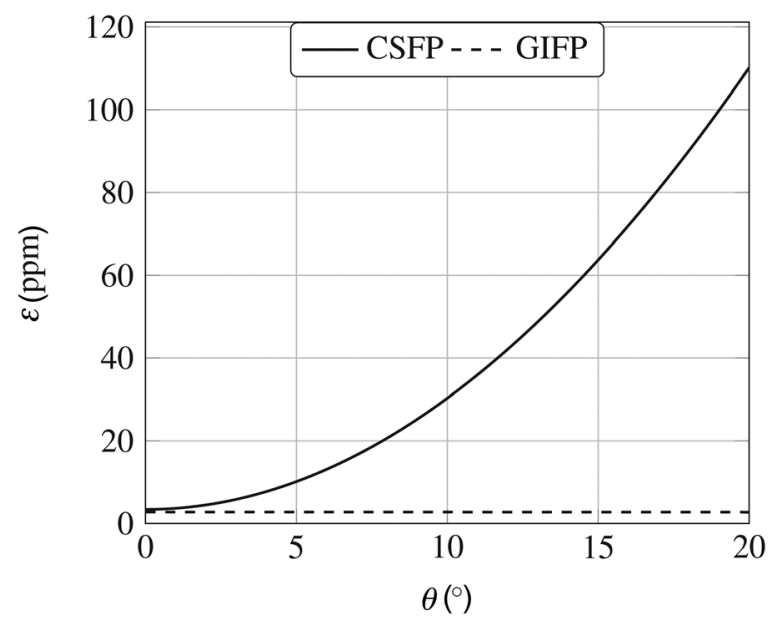

Fig. 10 Gravity sensitivity $\varepsilon$ of CSFP and GIFP versus rotation angle $\theta$ for normalized gravity load $\bar{N}=0.2$ and geometric parameter $\delta_{g}=\delta_{c}=0.127$ 


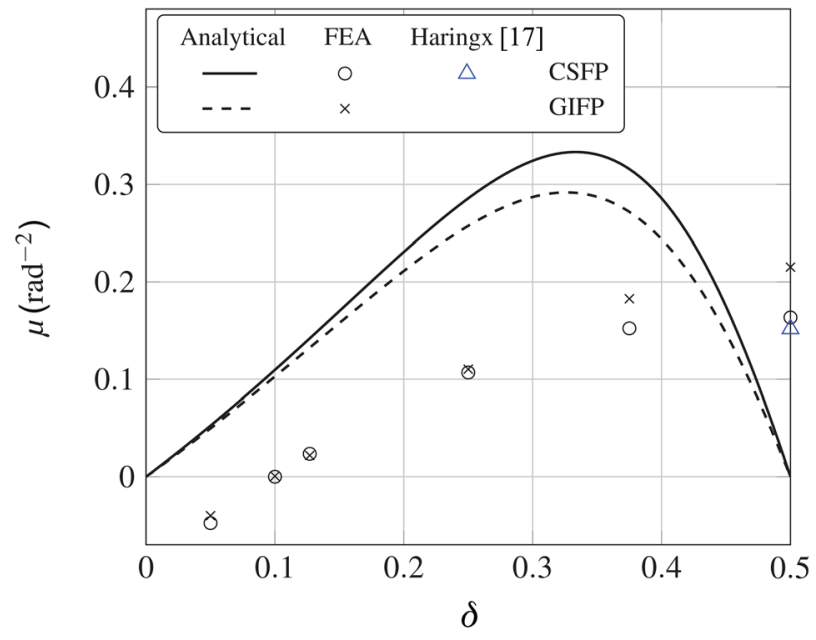

Fig. 11 Relative nonlinearity $\mu$ of CSFP and GIFP versus their geometric parameter $\delta$. Analytical and FEA results are shown.

where $\nu$ is the Poisson's ratio and we assume identical rods with circular cross sections for GIFP.

The relative nonlinearities of CSFP and GIFP $\mu$ are depicted in Fig. 11 versus their geometric parameter $\delta$. The FEA result for each $\delta$ is obtained by fitting an odd cubic polynomial to the torque-angle relationship. The relative nonlinearity is the ratio of the coefficient of the third-degree term to the coefficient of the first-degree term.

To validate our FEA, we use the analytical solution derived by Haringx for the nonlinear torque-angle relationship of CSFP with $\delta_{c}=0.5$, see Ref. [18, Eq. (37)]. Figure 11 shows that there is a good match between our FEA results and the relative nonlinearity extracted from Haringx's model. Note that Haringx's analytical model is limited to CSFP with $\delta_{c}=0.5$ since he used the inherent symmetry which holds only for this CSFP in order to solve the nonlinear equations. Hence, we are able to validate our FEA results only for $\delta_{c}=0.5$.

Figure 11 shows that there is not a good match between the relative nonlinearity obtained by our analytical model in Eq. (13) and the FEA results. The reason is that our analytical model does not take into account all the nonlinearities of the beams. There are three main sources of nonlinearity, only one of them is taken into account by our analytical model for nonlinearity:

(1) At the mobile tip of the beams, the bending moment and shear force are nonlinear functions of the bending angle and deflection which leads to nonlinearity of the beam stiffness. Our analytical model does not take this nonlinearity into account, i.e., we assume that the bending moment and shear force are linear functions of the bending angle and deflection, see Eq. (1).

(2) At the mobile tip of the beams, the deflection $(d \sin \theta$, see Eq. (3)) and the length of the lever arm of the shear force $(d \cos \theta$, see Eq. (3)) are nonlinear functions of the rotation angle $\theta$, which leads to stiffness nonlinearity. Our analytical model takes this nonlinearity into account.

(3) The pivot center of rotation $\mathrm{O}$ undergoes a parasitic shift during rotation which affects the stiffness of our pivots. The effect of parasitic shift is neglected in our analytical model of nonlinearity. However, the parasitic shift is taken into account in our analytical model of gravity sensitivity since gravity sensitivity is caused by the work of gravity force along the parasitic shift.

The FEA values are more reliable since they are validated by Haringx's analytical model of CSFP with $\delta_{c}=0.5$ [17]. Thus, we use the FEA results to compare the pivots. Our FEA results show

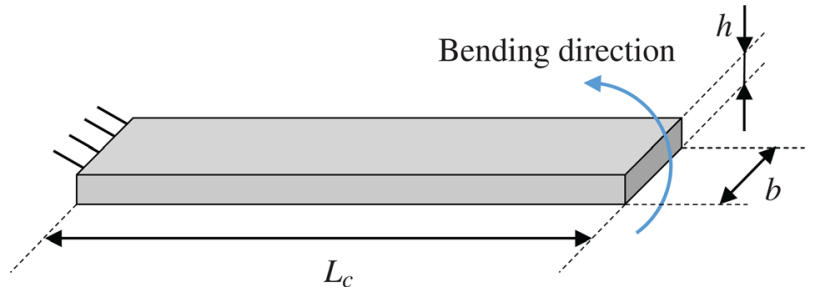

Fig. 12 Geometry of a cantilever beam with rectangular cross section

that for $\delta=0.1$, CSFP and GIFP nonlinearities become zero, achieving goal 2 of Sec. 1.2.

5.3 Stroke. For CSFP, rotation of the pivot gives rise to bending stress in the beams. However, for GIFP, it gives rise to not only bending stress in the codifferential beams but also torsional shear stress in the torsional beam. Hence, the stroke of CSFP is limited only by the bending stress of its beams whereas the stroke of GIFP is limited by either the bending stress of its codifferential beams or the torsional shear stress of its torsional beam.

In this section, we first derive the stroke of CSFP and GIFP based on the bending stress in their beams, which we call bending stroke. We then derive the stroke of GIFP based on the torsional shear stress of its torsional beam, which we call torsional stroke. We show that in our case where GIFP has five identical beams, its stroke is not limited by the shear stress of the torsional beam, but only by the bending stress of the codifferential beams.

5.3.1 Bending Stroke. Based on Refs. [6] and [11, Formula (2.3)], we derive the following formula for the bending stroke $S_{b}$ of CSFP and GIFP:

$$
S_{b}=\frac{1}{1+3|1-2 \delta|} \frac{2 \sigma_{\mathrm{adm}}}{E \alpha}
$$

where $\sigma_{\text {adm }}$ is the maximum admissible von Mises stress of the beams [11] and $\alpha$ is the aspect ratio of the beams. Note that $\delta$ stands for the geometric parameter of CSFP and GIFP. For the leaf-spring beams of CSFP having rectangular cross sections of width $b$ and thickness $h$, the aspect ratio is $\alpha=h / L_{c}$, see Fig. 12 . For GIFP having circular cross section rods of diameter $D$, the aspect ratio is $\alpha=D / L_{g}$. As given in Sec. 1.2, we are seeking the maximum stroke for given values of $\sigma_{\text {adm }}, \alpha$, and $E$. Formula (14) shows that the maximum stroke is achieved when $\delta=0.5$, while $\delta=0$ gives the minimum stroke.

Based on Eq. (14), we normalize the bending stroke

$$
S_{b}^{*}=\frac{E \alpha S_{b}}{2 \sigma_{\mathrm{adm}}}
$$

The normalized bending stroke of CSFP and GIFP versus their geometric parameter $\delta$ is depicted in Fig. 13. The analytical and FEA results match well with a slightly lower stroke for the latter. Note that, according to FEA results, $S_{b}^{*}(0.1)=0.29$, $S_{b}^{*}(0.127)=0.30$, and $S_{b}^{*}(0.5)=0.86$, so $S_{b}^{*}$ at $\delta=0.5$ is 2.9 times the value at $\delta=0.127$ and 3 times the value at $\delta=0.1$.

5.3.2 Torsional Stroke. The stroke of GIFP can be limited by the admissible stroke of its torsional beam. Based on Ref. [11, Formula (3.6)], we derive the following formula for the torsional stroke $S_{a}$ of a torsional beam with diameter $D$ :

$$
S_{a}=\frac{4(1+\nu) \sigma_{\mathrm{adm}}}{\sqrt{3} E \alpha} \frac{L_{a}}{L_{g}}
$$

Remark 5.3.2. As long as $S_{a}>S_{b}$, the stroke of GIFP is not limited by the shear stress in the torsional beam and Eq. (14) holds. 


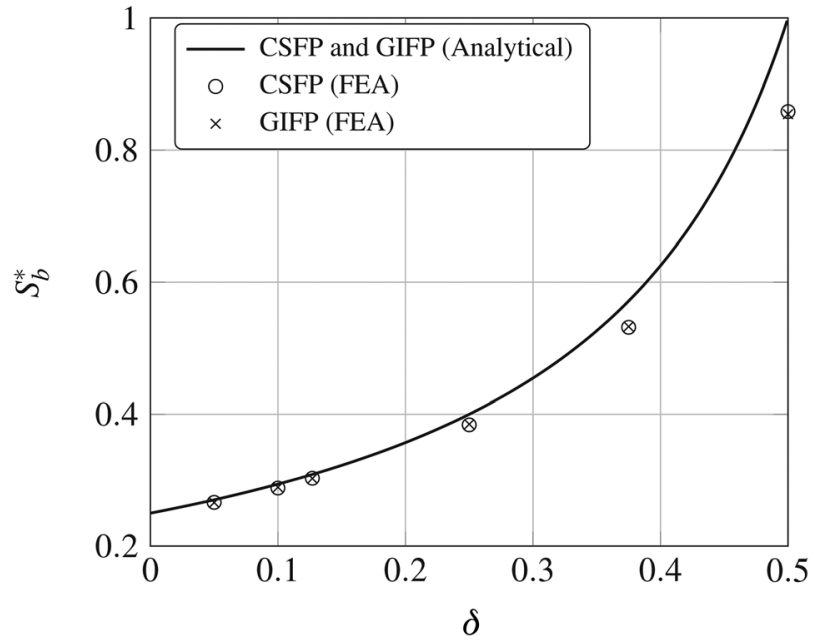

Fig. 13 Normalized bending strokes $S_{b}^{*}$ of CSFP and GIFP versus geometric parameter $\delta$. Analytical and FEA results are shown. The maximum admissible stress of the beams in the FEA was chosen such that the angle of the pivot stays below 20 deg.

This condition is satisfied for all values of $\delta$ when $L_{a} / L_{g}>\sqrt{3} / 2(1+\nu)$. Note that for our GIFP, where $\nu=0.3$ and $L_{a} / L_{g}=1$ (five identical rods), the condition is satisfied. So in this paper, wherever we referred to stroke, we meant the bending stroke given by Eq. (14).

\section{Conclusion}

This paper investigates the three crucial features of flexure pivots for their application to mechanical watch time bases. Analytical results are derived and validated numerically. We analyzed the well-known CSFP as a benchmark and showed the importance of the crossing point of its flexure beams for obtaining the desired features. We showed that CSFP can achieve only one of the three features at a time. We invented a new flexure pivot we call the GIFP based on a new flexure element we named, the codifferential. We showed that GIFP can, in principle, achieve two of the three desired features simultaneously, so is superior to CSFP.

Further research will involve analytical and numerical models of the thermal effect on the period of oscillations, miniaturization, and fabrication at the watch scale. We will also perform experiments to assess the effect of gravity, stroke, and temperature on the period of oscillations.

The codifferential concept is not limited to the application in this paper and has potential applications to other fields where insensitivity to gravity orientation plays a crucial role.

\section{References}

[1] Privat-Deschanel, P., and Focillon, A., 1877, Dictionnaire Général Des Sciences Techniques Et Appliquées, Garnier Frères, Paris, France.

[2] Barrot, F., Dubochet, O., Henein, S., Genequand, P., Giriens, L., Kjelberg, I., Renevey, P., Schwab, P., Ganny, F., and Hamaguchi, T., 2014, "Un Nouveau Régulateur Mécanique Pour une Réserve de Marche Exceptionnelle," Actes de la Journée d'Etude de la Société Suisse de Chronométrie, pp. 43-48.

[3] Bateman, D. A., 1977-1978, "Vibration Theory and Clocks," Horological J., 120-121(Pt. 7).

[4] Eastman, F. S., 1935, Flexure Pivots to Replace Knife Edges and Ball Bearings, an Adaptation of Beam-Column Analysis (Experiment Station series), University of Washington, University of Washington, Seattle, WA.

[5] Eastman, F. S., 1937, "The Design of Flexure Pivots," J. Aeronaut. Sci., 5(1), pp. 16-21.

[6] Wittrick, W. H., 1951, "The Properties of Crossed Flexure Pivots, and the Influence of the Point at Which the Strips Cross," Aeronaut. Q., 2(4), pp. 272-292.

[7] Henein, S., and Kjelberg, I., 2015, "Timepiece Oscillator," Swiss Center for Electronics and Microtechnology, Neuchâtel, Switzerland, U.S. Patent No. 9207641B2.

[8] Kahrobaiyan, M., Rubbert, L., Vardi, I., and Henein, S., 2016, "Gravity Insensitive Flexure Pivots for Watch Oscillators," Actes du Congrès International de Chronométrie, Montreux, Switzerland, pp. 49-55.

[9] Hongzhe, Z., Dong, H., and Shusheng, B., 2017, "Modeling and Analysis of a Precise Multibeam Flexural Pivot," ASME J. Mech. Des., 139(8), p. 081402 .

[10] Merriam, E. G., and Howell, L. L., 2016, "Lattice Flexures: Geometries for Stiffness Reduction of Blade Flexures," Precis. Eng., 45, pp. 160-167.

[11] Cosandier, F., Henein, S., Richard, M., and Rubbert, L., 2017, The Art of Flexure Mechanism Design, EPFL Press, Lausanne, Switzerland.

[12] ANSYS, 2017, "ANSYS ${ }^{\circledR}$ Workbench, Release 18.1, ANSYS Workbench User's Guide," ANSYS, Inc., Canonsburg, PA.

[13] Awtar, S., Slocum, H., and Sevincer, E., 2007, "Characteristics of Beam-Based Flexure Modules," ASME J. Mech. Des., 129(6), pp. 625-639.

[14] Plainevaux, J. E., 1956, "Etude des Déformations d'une Lame de Suspension Élastique," Nuovo Cimento, 4(4), pp. 922-928.

[15] Hongzhe, Z., and Shusheng, B., 2010, "Stiffness and Stress Characteristics of the Generalized Cross-Spring Pivot," Mech. Mach. Theory, 45(3), pp. 378-391.

[16] Hongzhe, Z., and Shusheng, B., 2010, "Accuracy Characteristics of the Generalized Cross-Spring Pivot,” Mech. Mach. Theory, 45(10), pp. 1434-1448.

[17] Graham, R. L., Knuth, D. E., and Patashnik, O., 1989, Concrete Mathematics, Addison Wesley, Reading, MA.

[18] Haringx, J. A., 1949, "The Cross-Spring Pivot as a Constructional Element," Appl. Sci. Res., 1(1), pp. 313-332. 